\title{
BOREL MEASURABILITY IN LINEAR ALGEBRA
}

\author{
EDWARD A. AZOFF
}

\begin{abstract}
It is shown that the usual processes of linear algebra (e.g., finding Jordan forms, eigenvalues, and eigenvectors) can be carried out in a Borel measurable fashion. These results follow easily from a variant of von Neumann's principle of measurable choice and can be applied to the study of Type $I_{n}$ von Neumann algebras.
\end{abstract}

Introduction. Denote by $M_{n}$ the algebra of $n \times n$ (complex) matrices, considered as operating on the Hilbert space $C^{n}$. We equip $M_{n}$ with its usual (operator) norm, along with the subordinate topological and Borel structures.

The main purpose of this paper is to show that many of the processes of linear algebra (e.g., finding Jordan canonical forms) are Borel measurable. The proofs, which turn out to be remarkably simple, depend only on a 'principle of measurable choice' presented as Theorem 1.

The considerations of the preceding paragraph turn out to be important in the study of Type $\mathrm{I}_{n}$ von Neumann algebras. (This is because such algebras can always be represented as algebras of matrices whose entries are continuous functions.) Indeed, upon examining the papers listed in the bibliography, one finds many arguments which are essentially measuretheoretic in nature. The latter half of the present paper shows how such arguments can be replaced by applications of Theorem 1 .

Measurable choice in linear algebra. It is easy to see that many of the processes of linear algebra are not continuous. For example, let $A_{n}=\left[\begin{array}{cc}0 & 1 / n \\ 0 & 0\end{array}\right]$. Then the $\left\{A_{n}\right\}_{n=1}^{\infty}$ converge uniformly to the zero matrix but the Jordan canonical form of each $A_{n}$ is $\left[\begin{array}{ll}0 & 1 \\ 0 & 0\end{array}\right]$. Thus the correspondence, $A \rightarrow$ Jordan form of $A$, is not continuous. Similar examples show that $\#(A)$, the number of distinct eigenvalues of $A$, does not depend continuously on $A$, and that there is no continuous way of associating an eigenvector with each matrix.

Received by the editors May 16, 1973.

AMS (MOS) subject classifications (1970). Primary 15A54, 28A20; Secondary $15 \mathrm{~A} 21,47 \mathrm{C} 15$.

Key words and phrases. Borel function, linear algebraic process, principle of measurable choice, continuous matrix valued function on a Stonian space.

(c) American Mathematical Society 1974 
The remainder of this section is devoted to showing that the processes of the preceding paragraph can be carried out in a Borel measurable fashion. The main tool in this development is provided by the following principle of measurable choice.

THEOREM 1. Let $X$ and $Y$ be complete separable metric spaces and $E$, a closed $\sigma$-compact subset of $X \times Y$. Then $\pi_{1}(E)$ is a Borel set in $X$ and there exists a Borel function $\varphi: \pi_{1}(E) \rightarrow Y$ whose graph is contained in $E$.

Proof. Let $F$ be a closed subset of $Y$. Then $E \cap X \times F$ is closed and hence can be expressed as a countable union of compact sets. The natural projection $\pi_{1}$ being continuous, we see that $\pi_{1}(E \cap X \times F)$ is the countable union of compact sets and hence is Borel. In particular, this applies to $F=Y$, whence $\pi_{1}(E)$ is Borel.

Fix a dense subset $\left\{y_{n}\right\}_{n=1}^{\infty}$ in $Y$. For each $x \in \pi_{1}(E)$, we define $\varphi_{k}(x)$ (inductively on $k$ ) to be the first $y_{n}$ satisfying

(1) $E$ is not disjoint from $\{x\} \times$ [closed $1 / 2^{k}$-ball about $y_{n}$ ], and

(2) either $k=1$ or the distance between $y_{n}$ and $\varphi_{k-1}(x)$ is $\leqq 1 / 2^{k-2}$.

An easy induction argument shows the functions $\left\{\varphi_{k}\right\}_{k=1}^{\infty}$ are all Borel. Thus, their (uniform) limit $\varphi$ satisfies the conclusion of the theorem.

REMARK. Theorem 1 is essentially a 'folk result'; in particular it follows from Theorem 4 (p. 135) and Exercise 18a (p. 149) of [1].

Theorem 1 differs from the usual von Neumann clipping theorem (see e.g., p. 35 of [2]) in that no set of measure zero must be deleted from $\pi_{1}(E)$.

COROLlaRY 2. There is a Borel function $\psi: M_{n} \rightarrow M_{n}$ associating with each matrix $A$, a unitary matrix $\psi(A)$ such that $\psi(A)^{*} A \psi(A)$ is upper triangular.

Proof. Let $E=\left\{(A, U, B) \in M_{n} \times M_{n} \times M_{n} \mid U\right.$ is unitary, $B$ is upper triangular and $\left.U^{*} A U=B\right\}$. Clearly $E$ is closed and since any matrix is unitarily equivalent to a matrix in upper triangular form, $\pi_{1}(E)=M_{n}$. Apply Theorem 1 with $X=M_{n}$ and $Y=M_{n} \times M_{n}$ to get a Borel function $\varphi: M_{n} \rightarrow M_{n} \times M_{n}$. The proof is completed by setting $\psi=\pi_{1} \circ \varphi$.

COROLlaRY 3. There is a Borel function $\psi: M_{n} \rightarrow M_{n}$ such that for each matrix $A, \psi(A)$ implements the similarity between $A$ and its Jordan canonical form.

Proof. Fix an integer $k$, and set

$$
\begin{aligned}
E_{k}=\left\{(A, J, P) \in M_{n} \times M_{n} \times M_{n} \mid J\right. \text { is in Jordan form, } \\
\left.\qquad\|P\| \leqq k,\left\|P^{-1}\right\| \leqq k \text { and } P A P^{-1}=J\right\} .
\end{aligned}
$$


We now apply Theorem 1 exactly as in the previous corollary to get a Borel function $\psi_{k}: \pi_{1}\left(E_{k}\right) \rightarrow \pi_{3}\left(E_{k}\right)$.

Now $\bigcup_{k=1}^{\infty} \pi_{1}\left(E_{k}\right)=M_{n}$ since every matrix has a Jordan form. Thus we take

$$
\begin{aligned}
\psi(A) & =\psi_{1}(A) \quad \text { for } A \in \pi_{1}\left(E_{1}\right), \\
& =\psi_{k}(A) \quad \text { for } A \in \pi_{1}\left(E_{k}\right) \backslash \pi_{1}\left(E_{k-1}\right), k>1,
\end{aligned}
$$

and the corollary is proved.

Corollary 4. (1) $\mathscr{J}(A)$, 'the' Jordan canonical form of $A$, depends measurably and boundedly on $A$.

(2) Let $G_{k}$ (respectively $H_{k}$ ) be the set of matrices in $M_{n}$ which have $k$ eigenvalues repeated (respectively not repeated) according to multiplicity. Then the $\left\{G_{k}\right\}$ and $\left\{H_{k}\right\}$ are all Borel sets.

(3) There exist $k$ Borel functions $\lambda_{1}(A) \cdots \lambda_{k}(A): H_{k} \rightarrow C$ which together trace out the spectrum of $A$.

(4) There exist $k$ Borel functions $v_{1}(A) \cdots v_{k}(A): G_{k} \rightarrow C_{n}$ such that each $v_{i}(A)$ is an eigenvector for $A$ and every eigenvector of $A$ is a linear combination of $v_{1}(A) \cdots v_{k}(A)$.

Proof. Let $\psi$ be as in Corollary 3.

(1) $\mathscr{J}(A)=\psi(A) A[\psi(A)]^{-1}$ is a Borel function of $A$ since $\psi$ is Borel. Also $\|\mathscr{J}(A)\| \leqq 1+\sup \{$ eigenvalues of $A\} \leqq 1+\|A\|$.

(2), (3), (4). The eigenvalues and eigenvectors of $A$ can be read off from $\mathscr{J}(A)$ and $\psi(A)$.

Applications to $M_{n}(X)$. Let $(X, \mu)$ be a perfect Stonian space and denote by $M_{n}(X)$, the $C$-*algebra of continuous functions from $X$ to $M_{n}$. (The algebraic operations on $M_{n}(X)$ are defined pointwise and the norm is the sup norm: $\|A\| \equiv \sup _{t \in X}\|A(t)\|$.) The algebras $M_{n}(X)$ have been widely studied (see references); their importance lies in the fact [7] that (to within unitary equivalence) they represent all homogeneous Type $I_{n}$ von Neumann algebras.

To say that $(X, \mu)$ is perfect Stonian means that $C(X)=L_{\infty}(X)$, i.e., every equivalence class of bounded measurable functions on $X$ has a unique continuous representative. In particular, a subset $S$ of $X$ has full measure $(\mu(X \mid S)=0)$ if and only if $S$ is dense in $X$. These facts are systematically exploited in the study of $M_{n}(X)$. For example, let $A, B \in$ $M_{n}(X)$ and consider the problem of finding a $Y \in M_{n}(X)$ such that $A Y=B$. Thus we want 'pointwise solutions' $\{Y(t)\}_{t \in X}$ depending continuously on $t$. Suppose we could find pointwise solutions $\{\tilde{Y}(t)\}_{t \in X}$ depending boundedly and measurably on $t$. Then $\tilde{Y}$ has a unique continuous representative $Y$ and $A(t) Y(t)=B(t)$ for almost all $t$. Since two continuous functions agreeing on a dense subset must coincide, we see that $A Y=B$. 
Thus we do not have to put solutions together continuously-it suffices to assemble them boundedly and measurably. It is the brunt of Theorem 1 that even the measurability requirement may be dropped. lent :

Corollary 5. Let $A, B \in M_{n}(X)$ and $K \geqq 0$. The following are equiva-

(1) The equation $A Y=B$ has a solution (in $M_{n}(X)$ ) of norm $\leqq K$.

(2) For almost all $t \in X$, the equation $A(t) Y=B(t)$ has $a$ solution (in $M_{n}$ ) of norm $\leqq K$.

Proof. Clearly $(1) \Rightarrow(2)$. Thus assume (2). Let

$$
E=\left\{(A, B, Y) \in M_{n} \times M_{n} \times M_{n} \mid A Y=B,\|Y\| \leqq K\right\} .
$$

Applying Theorem 1 with $X=M_{n} \times M_{n}$ and $Y=M_{n}$, we get a Borel function $\varphi: \pi_{M_{n} \times M_{n}}(E) \rightarrow M_{n}$. Set

$$
\begin{aligned}
\tilde{Y}(t) & =\varphi(A(t), B(t)), & & \text { when this is defined, } \\
& =0, & & \text { otherwise. }
\end{aligned}
$$

Let $Y$ be the unique continuous representative of $\tilde{Y}$. Then $A(t) Y(t)=B(t)$ almost everywhere and hence everywhere.

The following two results occur as Theorem 2 of [4] and Theorem 1 of [3] respectively. The proofs, being almost identical to that of Corollary 5 , are omitted.

Corollary 6 (PeARCy). Let $A \in M_{n}(X)$. Then there exists a unitary operator $U \in M_{n}(X)$ such that $U^{*} A U$ is in upper triangular form.

Corollary 7 (Deckard-Pearcy). Let $A, B \in M_{n}(X)$ and $K \geqq 0$. The following are equivalent:

(1) There is an invertible operator $P \in M_{n}(X)$ satisfying $P A P^{-1}=B$, $\|P\| \leqq K,\left\|P^{-1}\right\| \leqq K$.

(2) For almost all $t$, there is an invertible operator $P(t) \in M_{n}$ satisfying $P(t) A(t)[P(t)]^{-1}=B(t),\|P(t)\| \leqq K,\left\|[P(t)]^{-1}\right\| \leqq K$.

As a final application of Theorem 1, we give a simple proof of Theorem 4.6 of [7]. This result plays a central role in [5] and [7]. Recall that $R \in M_{n}(X)$ is said to be quasi-invertible if the only $D \in M_{n}(X)$ satisfying $R D=0$ or $D R=0$ is $D=0$. Two matrices $A, B \in M_{n}(X)$ are said to be quasi-similar if there exist quasi-invertible matrices $R$ and $S$ in $M_{n}(X)$ satisfying (1) $S A=B S$ and (2) $A R=R B$.

Corollary 8 (Hoover). Let $A \in M_{n}(X)$. There exists a $J \in M_{n}(X)$ such that $J(t)$ is in Jordan canonical form for each $t$ and $A$ is quasi-similar to $\mathrm{J}$. 
Proof. Let $\psi$ and $\mathscr{J}$ be as in Corollaries 3 and 4 respectively. Set

$$
\tilde{S}(t)=\frac{\psi(A(t))}{\|\psi(A(t))\|}, \quad \tilde{R}(t)=\frac{[\psi(A(t))]^{-1}}{\left\|[\psi(A(t))]^{-1}\right\|}, \quad \tilde{J}(t)=\mathscr{J}(A(t)),
$$

and denote their continuous representatives by $S, R, J$ respectively. We have for almost all $t$ :

(1) $J(t)$ is in Jordan form,

(2) $S(t) A(t)=J(t) S(t)$, and

(3) $A(t) R(t)=R(t) J(t)$.

The matrices involved being continuous, these statements must hold for all $t$. Moreover, $S(t)$ and $R(t)$ being invertible for almost all $t$, we see that $S$ and $R$ are quasi-invertible.

ADDED IN PROOF. If $X$ is merely assumed to be a Stonian space (i.e., not equipped with a measure) then every Borel function on $X$ differs from a continuous function on a set of first category. Thus Corollaries 5-8 have obvious analogues in this setting.

\section{REFERENCES}

General

1. N. Bourbaki, Eléments de mathématique. I: Les structures fondamentales de l'analyse. Fasc. VIII. Livre III: Topologie générale. Chap. 9: Utilisation des nombres réels en topologie générale, 2nd ed., Actualités Sci. Indust., no. 1045, Hermann, Paris, 1958; English transl., Hermann, Paris; Addison-Wesley, Reading, Mass., 1966. MR 30 \#3439; 34 \#5044b.

2. J. T. Schwartz, $W^{*}$-algebras, Gordon and Breach, New York, 1967. MR 38 \#547.

$M_{n}(X)$

3. D. Deckard and C. Pearcy, On continuous matrix-valued functions on a Stonian space, Pacific J. Math. 14 (1964), 857-869. MR 30 \#2356.

4. - On matrices over the ring of continuous complex-valued functions on a Stonian space, Proc. Amer. Math. Soc. 14 (1963), 322-328. MR 26 \#5438.

5. N. Dunford, A spectral theory for certain operators on a direct sum of Hilbert spaces, Math. Ann. 162 (1965/66), 294-330. MR 32 \#8182.

6. H. Gonshor, Spectral theory for a class of non-normal operators, Canad. J. Math. 8 (1956), 449-461. MR 18, 915.

7. T. Hoover, Hyperinvariant subspaces for n-normal operators, Acta. Sci. Math. (Szeged) 32 (1971), 109-119.

8. H. Radjavi and P. Rosenthal, Hyperinvariant subspaces for spectral and n-normal operators, Acta. Sci. Math. (Szeged) 32 (1971), 121-125.

See also references $1,7,9$, and 10 in [3].

Department of Mathematics, University of Georgia, Athens, Georgia 30601 